\title{
Distributionally Robust Appointment Scheduling with Moment-Based Ambiguity Set
}

\author{
Yiling Zhang* $\quad$ Siqian Shen ${ }^{\dagger} \quad$ S. Ayca Erdogan ${ }^{\ddagger}$ \\ January 25, 2017
}

\begin{abstract}
We study appointment scheduling under random service duration with unknown distributions. Given a sequence of appointments arriving at a single server, we assign their planned arrival time to minimize the expected total waiting time, while using a chance constraint to restrict the probability of server overtime. We consider a distributionally robust formulation based on an ambiguity set that uses the first two moments, and derive an approximate semidefinite programming model. We conduct computational studies by testing outpatient treatment scheduling instances.
\end{abstract}

Key words: appointment scheduling; random service durations; distributionally robust optimization; chance-constrained programming; semidefinite programming

\section{Introduction}

This paper studies the problem of scheduling a set of appointments with a fixed order of arrivals on a single server. The service durations are random and may be correlated. We assign each appointment an arrival time and minimize the expected total waiting time of all the appointments, subject to constrained risk of having server overtime. The traditional stochastic optimization methods require full knowledge of the distribution of uncertain parameters. We employ an ambiguity set of the unknown probability distribution function based on the first and the second moment information. We study a distributionally robust (DR) formulation that minimizes the worst-case (i.e., maximum) expected waiting time over the ambiguity set, and limits the worst-case overtime risk over the same ambiguity set.

Scheduling under uncertainty has been considered for many applications and the related problems are solved by using simulation, optimization, and approximation algorithms [see, e.g., 1, 2, 9, 12, 16, 20]. A common goal is to balance appointment waiting,

*Department of Industrial and Operations Engineering, University of Michigan at Ann Arbor, MI, USA;

${ }^{\dagger}$ Corresponding author, Department of Industrial and Operations Engineering, University of Michigan at Ann Arbor, MI, USA; email: siqian@umich.edu.

${ }^{\ddagger}$ Department of Industrial and Systems Engineering, San Jose State University, San Jose, CA, USA; 
server idleness, and server overtime. Some studies also optimize the sequence of appointments in addition to scheduling their arrival time $[7,8,18,19,20]$. The stochastic optimization literature often assume known distributions of the random service durations [1, 7, 10]. On the other hand, Epstein et al. [9], Mittal et al. [20] focus on robust scheduling model variants and seek "universal" scheduling/sequencing decisions under different uncertain cost structures. We refer to [11] for a thorough review of robust optimization approaches and relevant robust scheduling applications, [21] for a survey of scheduling theories and applications, and $[3,14]$ for comprehensive reviews of healthcare scheduling.

Meanwhile, DR approaches have been developed to address the issue of distributional ambiguity in the traditional stochastic programs, by utilizing statistical information of data samples for constructing ambiguity sets of the unknown distributions. We refer to $[4,24]$ for the representative work that uses moment-based ambiguity sets for optimizing DR expectation-based or chance-constrained models. In particular, by using an ambiguity set based on the first two moments, Jiang and Guan [15] successfully reformulate DR chance constraints as semidefinite programs (SDPs) based on conic duality.

The issue of distributional ambiguity has received increasing attention in the recent stochastic appointment scheduling literature. For example, both Kong et al. [17] and Mak et al. [19] consider DR scheduling problems, and minimize the worst-case expected waiting time of appointments and server overtime. In particular, Kong et al. [17] consider a cross-moment ambiguity set and derive a copositive programming reformulation. Mak et al. [19] derive a second-order conic program by only using marginal moments in the ambiguity set. We refer to $[5,6,22]$ for other recent papers that formulate DR models for server planning and /or appointment scheduling by using either moment- or densitybased ambiguity sets. Based on the structures of the related reformulations, they discuss continuous or discrete optimization methods for computing the optimal results.

In this problem, we optimize a DR objective that concerns the worst-case expected waiting time, subject to a DR chance constraint for restricting the server overtime. The ambiguity sets in both DR subproblems follow the moment-based form discussed in [15]. Different from the previous work, our model incorporates the distributional ambiguity and worst-case analysis in both the objective function of waiting time and the chance constraint of overtime. As the main technical contribution, we utilize special dual structures of the scheduling constraints and also the techniques in [15] to reformulate an SDP approximation of the DR model, which can be solved directly by off-the-shelf solvers. The computational efficacy of our approach depends on the efficiency of solving general continuous SDPs. We demonstrate the effect of distributional ambiguity by comparing the results of our SDP model with the ones of a sampling-based stochastic program on smallscale instances of outpatient treatment scheduling (involving six appointments). One can generalize the study in this paper to broader service optimization problems with similar structures, e.g., DR inventory control under random demand with unknown distributions [18].

The rest of the paper is organized as follows. Section 2 formulates the DR chanceconstrained scheduling problem and specifies the ambiguity set. Section 3 derives a conservative SDP approximate model of the DR problem. In Section 4, we demonstrate the computational results and solution patterns given by the DR approach, and compare 
them with those of the benchmark stochastic program based on discrete samples of outpatient treatment planning data.

Assumptions and Notation. We use $|X|$ to denote the cardinality of set $X$, and use $X \cdot Y$ to denote the Frobenius inner product of $X$ and $Y$, i.e., $X \cdot Y=\operatorname{tr}\left(X^{\top} Y\right)$. We denote $\mathbb{S}_{+}^{K}$ as the set of symmetric positive semidefinite $K \times K$ matrices. The generalized inequality for symmetric matrices, $X \succeq Y$, where $X, Y \in \mathbb{S}_{+}^{K}$, means that $X-Y \in \mathbb{S}_{+}^{K}$. Similarly, $X \preceq Y$ means that $Y-X \in \mathbb{S}_{+}^{K}$.

\section{DR Appointment Scheduling with Chance-Constrained Overtime}

A set of appointments $1, \ldots, m$ sequentially arrive at a single server, for which we plan an arrival time of each appointment. This is equivalent to assigning time intervals $x_{j}$ between each arrival pair of appointment $j$ and appointment $j+1$, for all $j=1, \ldots, m-1$. Let $x=\left[x_{1}, \ldots, x_{m-1}\right]^{\top}$. We consider the feasible region:

$$
x \in X:=\left\{x: \sum_{j=1}^{m-1} x_{j} \leq T, x_{j} \geq 0, \forall j=1, \ldots, m-1\right\},
$$

where $T$ represents the time limit of operating the server. The service durations of the $m$ appointments are denoted by random vector $s=\left[s_{1}, \ldots, s_{m}\right]^{\top}$ where $s_{j}$ is the time of serving appointment $j, \forall j=1, \ldots, m$. Instead of knowing the exact distribution of random parameter $s$, we are given a set of data samples $\left\{s^{k}\right\}_{k=1}^{K}$, with estimated mean and covariance matrix given by $\mu_{0}^{s}=1 / K \sum_{k=1}^{K} s^{k}$ and $\Sigma_{0}^{s}=1 / K \sum_{k=1}^{K}\left(s^{k}-\mu_{0}^{s}\right)\left(s^{k}-\mu_{0}^{s}\right)^{\top}$. Let $\Xi^{s}$ and $f^{s}$ be the support and the probability density function of the random parameter $s$, respectively. We consider the same ambiguity set $\mathcal{D}_{M}^{s}$ as the one used in $[4,15]$, given by

$$
\mathcal{D}_{M}^{s}=\mathcal{D}_{M}^{s}\left(\Xi^{s}, \mu_{0}^{s}, \Sigma_{0}^{s}, \gamma_{1}, \gamma_{2}\right)=\left\{\begin{array}{ll} 
& \int_{s \in \Xi^{s}} f^{s} d s=1 \\
f^{s}: & \left(\mathbb{E}[s]-\mu_{0}^{s}\right)^{\top}\left(\Sigma_{0}^{s}\right)^{-1}\left(\mathbb{E}[s]-\mu_{0}^{s}\right) \leq \gamma_{1} \\
& \mathbb{E}\left[\left(s-\mu_{0}^{s}\right)\left(s-\mu_{0}^{s}\right)^{\top}\right] \preceq \gamma_{2} \Sigma_{0}^{s}
\end{array}\right\} .
$$

The constraints in $\mathcal{D}_{M}^{s}$ ensures that (i) values of $f^{s}$ sum to 1 over the support $\Xi^{s}$; (ii) the mean of $s$ lies in an ellipsoid of size proportional to $\gamma_{1}$ centered at the empirical mean $\mu_{0}^{s}$; (iii) the true covariance matrix lies in a positive semidefinite cone bounded by a matrix inequality of $\gamma_{2} \Sigma_{0}^{s}$. We use parameters $\gamma_{1}$ and $\gamma_{2}$ to control the conservativeness of optimal solutions. In this paper, we set $\Xi^{s}=\mathbb{R}^{m}$.

Define $w_{j}$ as the waiting time variable of appointment $j$, for $j=1, \ldots, m$. Without loss of generality, appointment 1 always arrives at time 0 , and thus $w_{1}=0$. Let $h_{j}$ be a unit penalty cost of the waiting time of appointment $j$, for all $j=1, \ldots, m$. The goal is to minimize the maximum expected waiting penalty, while ensuring that the minimum probability of having no overtime is no less than $1-\alpha$. 
We formulate the DR chance-constrained scheduling problem as

$$
\begin{aligned}
& \text { [DRCC-S]: } \quad \min _{x \in X} \max _{f^{s} \in \mathcal{D}_{M}^{s}} \mathbb{E}_{f^{s}}\left[\min _{w} \sum_{j=2}^{m} h_{j} w_{j}\right] \\
& \text { s.t. } \quad w_{j}+x_{j-1} \geq s_{j-1}+w_{j-1}, \forall j=2, \ldots, m \\
& \inf _{f^{s} \in \mathcal{D}_{M}^{s}} \mathbb{P}\left(\sum_{j=1}^{m-1} x_{j}+w_{m}+s_{m} \leq T\right) \geq 1-\alpha \\
& w_{1}=0, w_{j} \geq 0, \forall j=2, \ldots, m,
\end{aligned}
$$

where the objective function (2a) minimizes the maximum expected cost of total waiting time for any distribution $f^{s} \in \mathcal{D}_{M}^{s}$. The arrival times of appointments $j-1$ and $j$ are $\sum_{i=1}^{j-2} x_{i}$ and $\sum_{i=1}^{j-1} x_{i}$, respectively, for all $j=2, \ldots, m$. The starting time of appointment $j$ is calculated as its arrival time plus possible waiting time of appointment $j$, i.e., $\sum_{i=1}^{j-1} x_{i}+$ $w_{j}$, which will be no earlier than completing appointment $j-1$, at time $\sum_{i=1}^{j-2} x_{i}+w_{j-1}+$ $s_{j-1}$. Canceling out $\sum_{i=1}^{j-2} x_{i}$ in the two terms, we have constraints (2b) hold for all $j=$ $2, \ldots, m$. The DR chance constraint (2c) enforces the worst-case (minimum) probability of finishing all the appointments before the time limit $T$ being no less than $1-\alpha$ for any $f^{s} \in \mathcal{D}_{M}^{s}$, in which the value of $\sum_{j=1}^{m-1} x_{j}+w_{m}+s_{m}$ represents the time of completing the last appointment $m$. The waiting time of appointment 1 is set to be zero and all the waiting times are nonnegative according to constraints (2d).

\section{Solution Methods for DRCC-S}

In this section, we develop reformulations of the DR objective (2a) and the DR chance constraint (2c), to solve DRCC-S as a monolithic model. The DRCC-S in model (2) involves recourse variables $w_{j}, \forall j=1, \ldots, m$, which we eliminate during the reformulation process and derive an approximated SDP that only involves continuous decision vector $x$.

\subsection{Reformulating the DR chance constraint (2c)}

A $w$-solution in constraint (2c) needs to satisfy constraints ( $2 \mathrm{~b})$ and $(2 \mathrm{~d})$, which are equivalent to $w_{1}=0$ and $w_{j}=\max \left\{0, s_{j-1}-x_{j-1}+w_{j-1}\right\}$, for $j=2, \ldots, m$. Recursively substituting $w_{j-1}$ in $w_{j}, j=2, \ldots, m$, we have

$$
w_{j}=\max _{k=1, \ldots, j-1}\left\{\sum_{i=k}^{j-1}\left(s_{i}-x_{i}\right), 0\right\}=\max _{k=1, \ldots, j}\left\{\sum_{i=k}^{j-1}\left(s_{i}-x_{i}\right)\right\},
$$

where the last equality follows the convention that $\sum_{i=j}^{j-1}\left(s_{i}-x_{i}\right)=0$.

We replace $w_{m}=\max _{k=1, \ldots, m}\left\{\sum_{i=k}^{m-1}\left(s_{i}-x_{i}\right)\right\}$ in the DR chance constraint (2c), yielding an equivalent DR joint chance constraint as follows.

$$
\inf _{f^{s} \in \mathcal{D}_{M}^{s}} \mathbb{P}\left(\sum_{j=1}^{m-1} x_{j}+\max _{k=1, \ldots, m}\left\{\sum_{i=k}^{m-1}\left(s_{i}-x_{i}\right)\right\}+s_{m}-T \leq 0\right) \geq 1-\alpha
$$




$$
\begin{aligned}
& \Leftrightarrow \quad \inf _{f^{s} \in \mathcal{D}_{M}^{s}} \mathbb{P}\left(\max _{k=1, \ldots, m}\left\{\sum_{i=k}^{m} s_{i}+\sum_{j=1}^{k-1} x_{j}-T\right\} \leq 0\right) \geq 1-\alpha \\
& \Leftrightarrow \quad \inf _{f^{s} \in \mathcal{D}_{M}^{s}} \mathbb{P}\left(\sum_{i=k}^{m} s_{i}+\sum_{j=1}^{k-1} x_{j}-T \leq 0, \forall k=1, \ldots, m\right) \geq 1-\alpha .
\end{aligned}
$$

We derive a reformulation of (3) by using a result studied in [15] for reformulating and solving general DR joint chance constraints.

Proposition 1. [15] The DR variant of a joint chance constraint in (3) can be approximated by a conservative SDP as:

$$
\begin{aligned}
& \cdot G+1-r+\Sigma_{0}^{s} \cdot H+\gamma_{1} q \leq \alpha \lambda \\
& {\left[\begin{array}{cc}
G & -p \\
-(p)^{\top} & 1-r
\end{array}\right] \succeq\left[\begin{array}{cc}
M & \frac{1}{2}\left(c+2 M \mu_{0}^{s}\right) \\
\frac{1}{2}\left(c+2 M \mu_{0}^{s}\right)^{\top} & \lambda+\left(\mu_{0}^{s}\right)^{\top} M \mu_{0}^{s}+c^{\top} \mu_{0}^{s}+d
\end{array}\right]} \\
& {\left[\begin{array}{cc}
M & \frac{1}{2}\left(c-\bar{a}_{k}\right) \\
\frac{1}{2}\left(c-\bar{a}_{k}\right)^{\top} & d+T-\sum_{j=1}^{k-1} x_{j}
\end{array}\right] \succeq 0 \quad \forall k=1, \ldots, m} \\
& \lambda \geq 0, r \in \mathbb{R}, M \in \mathbb{S}_{+}^{m \times m},\left[\begin{array}{cc}
G & -p \\
-(p)^{\top} & 1-r
\end{array}\right] \in \mathbb{S}_{+}^{(m+1) \times(m+1)},\left[\begin{array}{cc}
H & p \\
(p)^{\top} & q
\end{array}\right] \in \mathbb{S}_{+}^{(m+1) \times(m+1)}
\end{aligned}
$$

where $\bar{a}_{k}=[\underbrace{0, \ldots, 0}_{k-1}, \underbrace{1, \ldots, 1}_{m-k+1}]^{\top} \in \mathbb{R}^{m}$, for $k=1, \ldots, m$. (The elements in each $\bar{a}_{k}, k=$ $1, \ldots, m$ are coefficients of $s_{1}, \ldots, s_{m}$ in the DR chance constraint (3).)

We describe the key idea in [15] for proving Proposition 1 as follows. Denote a variable matrix $M \in \mathbb{S}_{+}^{m \times m}$, a variable vector $c \in \mathbb{R}^{m}$, and a variable scalar $d \in \mathbb{R}$. We can bound the piecewise linear function $\max _{k=1, \ldots, m}\left\{\sum_{i=k}^{m} s_{i}+\sum_{j=1}^{k-1} x_{j}-T\right\}$ from above by a quadratic function $s^{\top} M s+c^{\top} s+d$ for some appropriately assigned values of $M, c$, and $d$, such that

$$
\inf _{f^{s} \in \mathcal{D}_{M}^{s}} \mathbb{P}\left(s^{\top} M s+c^{\top} s+d \leq 0\right) \leq \inf _{f^{s} \in \mathcal{D}_{M}^{s}} \mathbb{P}\left(\max _{k=1, \ldots, m}\left\{\sum_{i=k}^{m} s_{i}+\sum_{j=1}^{k-1} x_{j}-T\right\} \leq 0\right) .
$$

Therefore, the DR chance constraint $\inf _{f^{s} \in \mathcal{D}_{M}^{s}} \mathbb{P}\left(s^{\top} M s+c^{\top} s+d \leq 0\right) \geq 1-\alpha$ implies the DR joint chance constraint (3) (or equivalently (2c)). Following conic duality, [15] show that (4a), (4b), and (4d) are equivalent to the above DR chance constraint with the quadratic function; (4c) ensures that the quadratic function is always above the piecewise linear function. As a result, the SDP model in Proposition 1 serves as a conservative approximation to (3) (and to (2c)), which indicates that its optimal solution can satisfy (2c), but could be too conservative such that the left-hand side of $(2 \mathrm{c})$ is satisfied at a probability level higher than $1-\alpha$. The details of the proof of Proposition 1 are given in [15]. 


\subsection{Reformulating the DR objective (2a)}

To reformulate the DR expectation-based objective (2a), we specify the inner problem of DRCC-S, for any given $x \in X$, as follows.

$$
\begin{aligned}
\max _{f^{s}} & \int_{s \in \Xi^{s}}\left(\min _{w \in \Gamma_{w}(x, s)} \sum_{j=2}^{m} h_{j} w_{j}\right) f_{s} d s \\
\text { s.t. } & \int_{s \in \Xi^{s}} f^{s} d s=1 \\
& \int_{s \in \Xi^{s}}\left(s-\mu_{0}^{s}\right)\left(s-\mu_{0}^{s}\right)^{\top} f^{s} d s \preceq \gamma_{2} \Sigma_{0}^{s} \\
& \int_{s \in \Xi^{s}}\left[\begin{array}{cc}
\Sigma_{0}^{s} & s-\mu_{0}^{s} \\
\left(s-\mu_{0}^{s}\right)^{\top} & \gamma_{1}
\end{array}\right] f^{s} d s \succeq 0,
\end{aligned}
$$

where in the objective (5a), $\Gamma_{w}(x, s):=\left\{w \in \mathbb{R}_{+}^{m}:(2 \mathrm{~b}),(2 \mathrm{~d})\right\}$ represents the set of feasible $w$-solutions that satisfy constraints (2b) and (2d). Constraints (5b), (5c) and (5d) are related to the three constraints in set $\mathcal{D}_{M}^{s}$ specified in (1). Model (5) computes the worst-case (maximum) expected total waiting time for any $f^{s}$ realization in the ambiguity set $\mathcal{D}_{M}^{s}$.

Denote $\chi_{i j}=\left\{\begin{array}{ll}0 & \text { if } 1 \leq i=j \leq m-1 \\ \sum_{l=i+1}^{j} h_{l} & \text { if } 1 \leq i<j \leq m-1\end{array}\right.$. For each $k, j$ such that $1 \leq k \leq j \leq$ $m-1$, let vector $\bar{a}^{k j}=[\underbrace{0, \ldots, 0}_{k-1}, \chi_{k j}, \chi_{(k+1) j}, \ldots, \chi_{j j}, \underbrace{0, \ldots, 0}_{m-1-j}]^{\top}$. We utilize the one-to-one correspondence [see 18,19] between partitions of the set $\{1, \ldots, m\}$ of appointments and extreme points of the feasible region of appointment scheduling, defined by (2b) and (2d), to derive equivalent convex reformulation of the inner problem (5).

Proposition 2. The inner problem (5) has the same optimal objective value as the one of the following SDP:

$$
\begin{aligned}
\min _{\varepsilon, G^{\prime}, H^{\prime}, p^{\prime}, q^{\prime}} & \sum_{i=1}^{m-1} \varepsilon_{i}+\gamma_{2}\left(\Sigma_{0}^{s} \cdot G^{\prime}\right)-\left(\mu_{0}^{s}\right)^{\top} G^{\prime} \mu_{0}^{s}+\Sigma_{0}^{s} \cdot H^{\prime}-2\left(\mu_{0}^{s}\right)^{\top} p^{\prime}+\gamma_{1} q^{\prime} \\
\text { s.t. } & {\left[\begin{array}{cc}
G^{\prime} & -\left(\frac{1}{2} \bar{a}^{k j}+p^{\prime}+G^{\prime} \mu_{0}^{s}\right) \\
-\left(\frac{1}{2} \bar{a}^{k j}+p^{\prime}+G^{\prime} \mu_{0}^{s}\right)^{\top} & \sum_{i=k}^{j} \varepsilon_{i}+\sum_{i=k}^{j} \chi_{i j} x_{i}
\end{array}\right] \succeq 0, \forall 1 \leq k \leq j \leq m-1, } \\
& G^{\prime} \succeq 0, G^{\prime} \in \mathbb{R}^{m \times m},\left[\begin{array}{cc}
H^{\prime} & p^{\prime} \\
\left(p^{\prime}\right)^{\top} & q^{\prime}
\end{array}\right] \succeq 0,\left[\begin{array}{cc}
H^{\prime} & p^{\prime} \\
\left(p^{\prime}\right)^{\top} & q^{\prime}
\end{array}\right] \in \mathbb{R}^{(m+1) \times(m+1) .}
\end{aligned}
$$

Proof. We employ conic duality [see 23] and a special structure in the dual problem of Model (5) to show the above result. First, we associate dual variables $r^{\prime} \in \mathbb{R}, G^{\prime} \in \mathbb{R}^{m \times m}$, and $\left[\begin{array}{cc}H^{\prime} & p^{\prime} \\ \left(p^{\prime}\right)^{\top} & q^{\prime}\end{array}\right] \in \mathbb{R}^{(m+1) \times(m+1)}$ with constraints (5b), (5c) and (5d), respectively. We formulate the dual of Model (5) as

$$
\begin{aligned}
\min _{G^{\prime}, H^{\prime}, p^{\prime}, q^{\prime}, r^{\prime}} & r^{\prime}+\gamma_{2}\left(\Sigma_{0}^{s} \cdot G^{\prime}\right)-\left(\mu_{0}^{s}\right)^{\top} G^{\prime} \mu_{0}^{s}+\Sigma_{0}^{s} \cdot H^{\prime}-2\left(\mu_{0}^{s}\right)^{\top} p^{\prime}+\gamma_{1} q^{\prime} \\
\text { s.t. } & r^{\prime} \geq \min _{w \in \Gamma_{w}(x, s)} \sum_{j=2}^{m} h_{j} w_{j}+2 s^{\top}\left(p^{\prime}+G^{\prime} \mu_{0}^{s}\right)-s^{\top} G^{\prime} s, \forall s \in \mathbb{R}^{m}
\end{aligned}
$$




$$
r^{\prime} \in \mathbb{R}, G^{\prime} \succeq 0,\left[\begin{array}{cc}
H^{\prime} & p^{\prime} \\
\left(p^{\prime}\right)^{\top} & q^{\prime}
\end{array}\right] \succeq 0 .
$$

We consider the minimization problem on the right-hand side of the constraint $(7 \mathrm{~b})$ :

$$
\min _{w}\left\{\sum_{j=2}^{m} h_{j} w_{j}:(2 \mathrm{~b}),(2 \mathrm{~d})\right\} \text {. }
$$

We associate dual variables $\delta_{i}, i=1, \ldots, m-1$ with constraints (2b), and formulate the dual of model (8) as

$$
\begin{aligned}
\max _{\delta} & \sum_{j=1}^{m-1} \delta_{j}\left(s_{j}-x_{j}\right) \\
\text { s.t. } & \delta_{j-1}-\delta_{j} \leq h_{j}, \forall j=2, \ldots, m-1 \\
& \delta_{m-1} \leq h_{m} \\
& \delta_{j} \geq 0, \forall j=1, \ldots, m-1 .
\end{aligned}
$$

We use $\Gamma_{\delta}:=\{\delta:(9 \mathrm{~b})-(9 \mathrm{~d})\}$ to denote the dual feasible region, which is independent on the decision $x$ and uncertainty $s$. According to [19], the values of extreme points of set $\Gamma_{\delta}$ have either $\delta_{m-1}=h_{m}$ or $\delta_{m-1}=0$, depending on whether $w_{m}=0$ or $w_{m}>0$, respectively, which result from the complementary slackness formed between the primal (8) and the dual (9). We use the value of $\delta_{m-1}$ as an incumbent solution, and rearrange inequalities in (9b) as $\delta_{j-1} \leq \delta_{j}+h_{j}, \forall j=2, \ldots, m-1$. Recursively, following the complementary slackness, the optimal value of $w_{j}$ determines that whether $\delta_{j-1}$ takes the upper bound value $\delta_{j}+h_{j}$ or the lower bound value 0 .

Then, there is a one-to-one correspondence between an extreme point of set $\Gamma_{\delta}$ and a partition of the set $\{1, \ldots, m-1\}$ into intervals. For each interval $\{k, \ldots, j\} \subset\{1, \ldots, m-$ $1\}$ in the partition, the corresponding extreme-point solution has $\delta_{j}=0$, and $\delta_{i}=\sum_{l=i+1}^{j} h_{l}$ for all $i=k, \ldots, j-1$. That is, for each interval $\{k, \ldots, j\}$ in the partition and $i \in\{k, \ldots, j\}$, the value of $\delta_{i}$ is given by $\chi_{i j}$, whose value was specified in Proposition 2.

Thus, define indicating binary variables $t_{k j}, \forall 1 \leq k \leq j \leq m-1$, such that $t_{k j}=1$ if interval $\{k, \ldots, j\}$ belongs to the partition (i.e., $t_{k j}=1$ if $\delta_{i}=\chi_{i j}$ ), and $t_{k j}=0$ otherwise. The variables $t_{k j}$ represent a valid partition if and only if each element $i$ only belongs to one interval, stated as

$$
\sum_{k=1}^{i} \sum_{j=i}^{m-1} t_{k j}=1, \forall i=1, \ldots, m-1 .
$$

Denote set $\Gamma_{t}=\left\{t_{k j}:(10), t_{k j} \geq 0, \forall 1 \leq k \leq j \leq m-1\right\}$, where we relax the binary variables $t_{k j}$ as continuous variables. Still, any extreme point of the set $\Gamma_{t}$ has binaryvalued $t_{k j}$, because the coefficient matrix in (10) has a consecutive-ones property and is thus totally unimodular.

Recall constraint (7b). We reformulate its right-hand side by the equivalent maximization model (9) following the strong duality, yielding:

$$
r^{\prime} \quad \geq \max _{s \in \mathbb{R}^{m}} \max _{\delta \in \Gamma_{\delta}} \sum_{i=1}^{m-1} \delta_{i}\left(s_{i}-x_{i}\right)+2 s^{\top}\left(p^{\prime}+G^{\prime} \mu_{0}^{s}\right)-s^{\top} G^{\prime} s
$$




$$
=\max _{\delta \in \Gamma_{\delta}} \max _{s \in \mathbb{R}^{m}} \sum_{i=1}^{m-1} \delta_{i}\left(s_{i}-x_{i}\right)+2 s^{\top}\left(p^{\prime}+G^{\prime} \mu_{0}^{s}\right)-s^{\top} G^{\prime} s .
$$

We can interchange the order of $\max _{\delta \in \Gamma_{\delta}}$ and $\max _{s \in \mathbb{R}^{m}}$, because the objective function is bounded with respect to $\delta$ and $s$. We form the relationship between the partitions and extreme points in $\Gamma_{\delta}$ with the above dual formulation. Moreover, constraint (11b) is equivalent to

$$
\max _{t \in \Gamma_{t}} \sum_{k=1}^{m-1} \sum_{j=k}^{m-1} \max _{s \in \mathbb{R}^{m}}\left(\sum_{i=k}^{j} \chi_{i j}\left(s_{i}-x_{i}\right)+2 s^{\top}\left(p^{\prime}+G^{\prime} \mu_{0}^{s}\right)-s^{\top} G^{\prime} s\right) t_{k j} .
$$

To convert problem (12) as a minimization problem, we associate dual variables $\varepsilon_{i}, i=$ $1, \ldots, m-1$ with the constraints in $\Gamma_{t}$, and formulate the dual problem as

$$
\begin{aligned}
\min _{\varepsilon} & \sum_{i=1}^{m-1} \varepsilon_{i} \\
\text { s.t. } & \sum_{i=k}^{j} \varepsilon_{i} \geq \sum_{i=k}^{j} \chi_{i j}\left(s_{i}-x_{i}\right)+2 s^{\top}\left(p^{\prime}+G^{\prime} \mu_{0}^{s}\right)-s^{\top} G^{\prime} s \forall s \in \mathbb{R}^{m}, \forall 1 \leq k \leq j \leq m-1 .(13
\end{aligned}
$$

Note that constraint (13b) is equivalent to $\sum_{i=k}^{j} \varepsilon_{i} \geq \max _{s} \sum_{i=k}^{j} \chi_{i j}\left(s_{i}-x_{i}\right)+2 s^{\top}\left(p^{\prime}+\right.$ $\left.G^{\prime} \mu_{0}^{s}\right)-s^{\top} G^{\prime} s, \forall s \in \mathbb{R}^{m}$, which can be written as an SDP

$$
\left[\begin{array}{cc}
G^{\prime} & -\left(\frac{1}{2} \bar{a}^{k j}+p^{\prime}+G^{\prime} \mu_{0}^{s}\right) \\
-\left(\frac{1}{2} \bar{a}^{k j}+p^{\prime}+G^{\prime} \mu_{0}^{s}\right)^{\top} & \sum_{i=k}^{j} \varepsilon_{i}+\sum_{i=k}^{j} \chi_{i j} x_{i}
\end{array}\right] \succeq 0, \forall 1 \leq k \leq j \leq m-1,
$$

with the values of $\bar{a}^{k j}$ specified in Proposition 2 , for each $k, j$ such that $1 \leq k \leq j \leq m-1$. Finally, combining (7a), (7c), (11b), (13a), and (14), we show that the SDP model (6) is equivalent to the inner problem (5), and thus complete the proof.

Next we combine the reformulation (6) with the outer minimization problem in the objective function (2a). According to Propositions 1 and 2, we can conclude the following result.

Theorem 1. The optimal solution to DRCC-S can be solved via a conservative SDP model

[R-DRCC-S]: $\min \{(6 \mathrm{a}):(4 \mathrm{a})-(4 \mathrm{~d}),(6 \mathrm{~b})-(6 \mathrm{c}), x \in X\}$.

\section{Computational Results}

We show the computational results for DRCC-S by solving R-DRCC-S, and compare them with the results of a sampling-based stochastic linear program that minimizes the expected penalty of overtime and waiting time [see, e.g., 7]. The benchmark model considers discrete samples of the random variable $s$, given by $s^{1}, \ldots, s^{K}$, where $K$ is the number of samples, and let $p_{k}$ be the probability that $s=s^{k}$, for all $k=1, \ldots, K$, such 
that $\sum_{k=1}^{K} p_{k}=1$. Given a scheduling solution $x$, both of the overtime and waiting time depend on the realization of the random service durations $s$, and therefore, we define $W^{k} \geq 0$ and $w_{j}^{k}, j=1, \ldots, m$ as auxiliary decision variables representing overtime of the server and waiting time of appointments, respectively, for each $k=1, \ldots, K$. We penalize the overtime by a unit cost $h_{W}$ and penalize each appointment $j$ 's waiting time by a unit cost $h_{j}, \forall j=1, \ldots, m$. We minimize the expected total penalty cost of waiting and overtime in the following benchmark model.

[Stoch-S]:

$$
\begin{aligned}
\min _{\substack{x \in X, w^{1}, \ldots, w^{K} \\
W^{1}, \ldots, W^{K}}} & \sum_{k=1}^{K} p_{k}\left(\sum_{j=2}^{m} h_{j} w_{j}^{k}+h_{W} W^{k}\right) \\
\text { s.t. } & w_{j}^{k}+x_{j-1} \geq s_{j-1}^{k}+w_{j-1}^{k}, \forall j=2, \ldots, m, \forall k=1, \ldots, K \\
& \sum_{j=1}^{m-1} x_{j}+w_{m}^{k}+s_{m}^{k}-W^{k} \leq T, \forall k=1, \ldots, K \\
& W^{k} \geq 0, w_{1}^{k}=0, w_{j}^{k} \geq 0, \forall j=2, \ldots, m, \forall k=1, \ldots, K,
\end{aligned}
$$

The constraints (15b), (15c), (15d) are generalized from the previous constraints (2b), (2c), (2d), respectively. They are specified for each sample $k$, for all $k=1, \ldots, K$, and compute the values of $w_{j}^{k}$ and $W^{k}$ dependent on the values of $x$ and $s^{k}$.

\subsection{Computational Setup}

We test $m=6$ appointments and set the operating time limit $T=8$ hours. We use $h_{j}=1$ for each appointment $j$ as the unit waiting time cost, and set $h_{W}=5$ as the unit overtime cost, used in both the DR model and the benchmark model. The computation consists of two phases: First, we compute optimal solutions of DRCC-S and Stoch-S by using a limited number of scenarios (i.e., training data); second, we fix the solutions in test data which involves a large number of scenarios sampled from the underlying true distribution. We consider an application of outpatient treatment planning, where the true distribution $f^{s}$ of the random service time $s$ often follows Lognormal [see 13], and we generate the training data based on two distributional types: Lognormal and Gamma, where the latter indicates a misspecification of the true distribution. In this paper, we use 20 scenarios for the training data and 10,000 scenarios for the test data. We generate the realized service duration of each appointment in the training/test data by following a given distribution type with both the mean value and standard deviation being 12.5 minutes in all the scenarios. For DRCC-S, we test two overtime risk levels: $\alpha=5 \%$ and $10 \%$. All computational tests are performed on a Windows 7 machine with Intel(R) Core(TM) i7-2600 CPU 3.40 GHz and 8GB memory. We solve all the models by implementing CVX in MATLAB with MOSEK as the optimization solver.

\subsection{Results and Solution Patterns}

We run the first phase of computation under Lognormal and Gamma distributed training data, and depict optimal solution patterns to DRCC-S with $\alpha=5 \%, 10 \%$, and to Stoch-S 
in Figure 1. For R-DRCC-S, we show the results for the case when $\left(\gamma_{1}, \gamma_{2}\right)=(1,2)$.

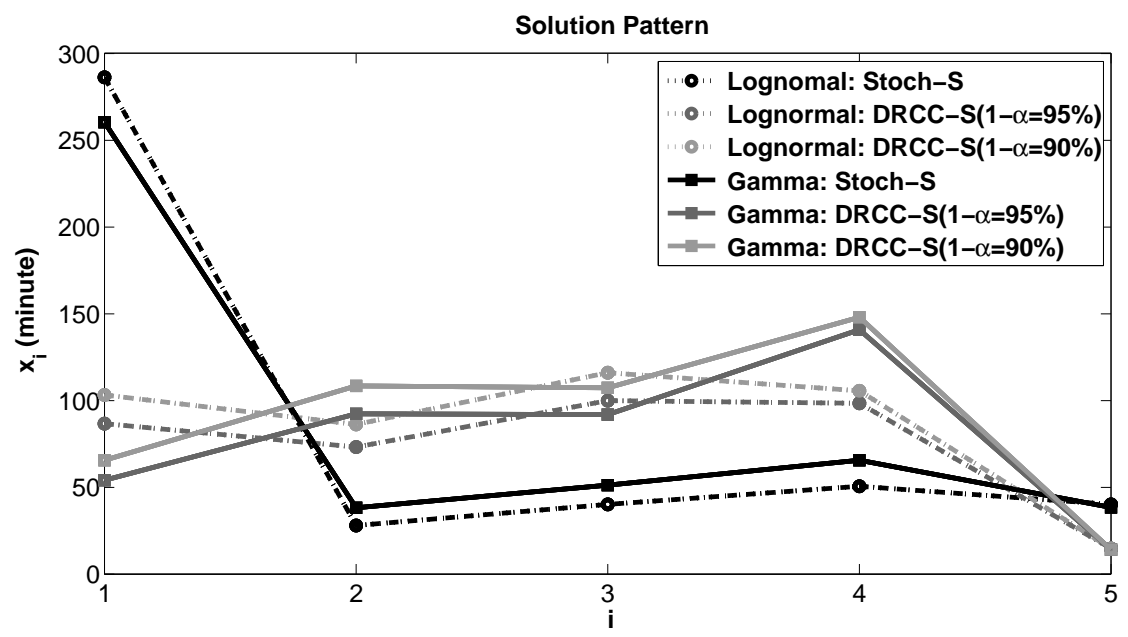

Figure 1: Solution patterns with $\left(\gamma_{1}, \gamma_{2}\right)=(1,2)$ using Lognormal and Gamma distributed data

We summarize the observations as follows. First, under limited data samples and distributional ambiguity, optimal scheduling solutions from the stochastic benchmark model do not demonstrate a "dome" shape as shown in the literature [7] when large scale data samples are available. (In a dome shape, the inter-arrival time is shorter for earlier and later appointments, and is longer for the middle ones.) Second, we are able to demonstrate an approximate "dome" shape of the DRCC-S solutions. As $1-\alpha$ increases in the DR constraint $(2 \mathrm{c})$, we observe decreased inter-arrival time to prevent overtime.

Next, we conduct the second phase of computation to evaluate the performance of solutions to DRCC-S and to Stoch-S in the test data with 10,000 scenarios. "DR(1,2)" and "DR(0,1)" in Table 1 indicate the results for R-DRCC-S with $\left(\gamma_{1}, \gamma_{2}\right)=(1,2)$ and $=(0,1)$, respectively. We compute the solution reliability as the percentage of "overtimefree" scenarios in the test data, and present the results for the cases when training data follows "Lognormal" or "Gamma". The reliability results associated with DR $(1,2)$ are all better than those given by Stoch-S. Specifically, when we solve the models with Gamma distributed training data, the reliability results become much better; meanwhile, $\operatorname{DR}(0,1)$ could perform worse than the desirable reliability $1-\alpha=90 \%$, due to that we use smaller $\gamma_{1}, \gamma_{2}$, and thus a smaller, more risk-seeking ambiguity set.

Table 1: Reliability of optimal solutions to DRCC-S and Stoch-S models

\begin{tabular}{clll}
\hline Approach & $1-\alpha$ & Lognormal & Gamma \\
\hline Stochastic & N/A & $94.44 \%$ & $89.29 \%$ \\
DR(1,2) & $95 \%$ & $99.67 \%$ & $99.30 \%$ \\
& $90 \%$ & $97.00 \%$ & $92.13 \%$ \\
DR(0,1) & $95 \%$ & $96.75 \%$ & $91.63 \%$ \\
& $90 \%$ & $73.84 \%$ & $24.80 \%$ \\
\hline
\end{tabular}

In Table 2, we also report the overtime and waiting time statistics based on the test data. The average waiting time given by Stoch-S solutions is shorter than that given by 
DRCC-S solutions, while the standard deviation of waiting time in the latter is smaller. Also, DRCC-S solutions provide shorter overtime, in terms of expectation, standard deviation, and $\geq 50 \%$ higher quantiles. This reflects that while trading off between appointment waiting and server overtime, the DR approach emphasizes more on ensuring shorter overtime with sufficiently high probability subject to unknown distributions.

Table 2: Overtime and waiting time statistics (in minute)

\begin{tabular}{cccccccc}
\hline & & Avg. & Std. & \multicolumn{4}{c}{ Quantiles } \\
& & & & $50 \%$ & $75 \%$ & $90 \%$ & $99 \%$ \\
\hline Stochastic & Wating time & 2.94 & 22.34 & 0.00 & 0.00 & 0.00 & 85.97 \\
& Overtime & 1.14 & 7.43 & 0.00 & 0.00 & 0.00 & 32.36 \\
\hline DR $(1,2)$ with Lognormal training data \\
with $1-\alpha=95 \%$ & Wating time & 3.66 & 18.84 & 0.00 & 0.63 & 10.38 & 49.53 \\
& Overtime & 0.12 & 3.09 & 0.00 & 0.00 & 0.00 & 0.00 \\
\hline
\end{tabular}

In our future research, we will incorporate appointment ordering decisions into the proposed DR model, and also compute larger-size instances by investigating more efficient solution methods.

Acknowledgement. The authors are grateful to the Associate Editor and anonymous reviewers for their helpful comments and suggestions. Dr. Shen acknowledges partial support by the National Science Foundation under grant grant CMMI-1433066.

\section{References}

[1] Begen, M. A. and Queyranne, M. (2011). Appointment scheduling with discrete random durations. Mathematics of Operations Research, 36(2):240-257.

[2] Berg, B. P., Denton, B. T., Erdogan, S. A., Rohleder, T., and Huschka, T. R. (2014). Optimal booking and scheduling in outpatient procedure centers. Computers $\mathcal{E}$ Operations Research, 50:24-37.

[3] Cayirli, T. and Veral, E. (2003). Outpatient scheduling in health care: a review of literature. Production and Operations Management, 12(4):519-549.

[4] Delage, E. and Ye, Y. (2010). Distributionally robust optimization under moment uncertainty with application to data-driven problems. Operations Research, 58(3):595-612.

[5] Deng, Y. and Shen, S. (2016). Decomposition algorithms for optimizing multi-server appointment scheduling with chance constraints. Mathematical Programming, 157(1):245-276.

[6] Deng, Y., Shen, S., and Denton, B. T. (2016). Chance-constrained surgery planning under conditions of limited and ambiguous data. Available at SSRN: http://dx.doi.org/10 . $2139 / \operatorname{ssrn} .2432375$.

[7] Denton, B. T. and Gupta, D. (2003). A sequential bounding approach for optimal appointment scheduling. IIE Transactions, 35(11):1003-1016.

[8] Denton, B. T., Viapiano, J., and Vogl, A. (2007). Optimization of surgery sequencing and scheduling decisions under uncertainty. Health Care Management Science, 10(1):13-24.

[9] Epstein, L., Levin, A., Marchetti-Spaccamela, A., Megow, N., Mestre, J., Skutella, M., and Stougie, L. (2012). Universal sequencing on an unreliable machine. SIAM Journal on Computing, 41(3):565-586. 
[10] Erdogan, S. A. and Denton, B. T. (2013). Dynamic appointment scheduling of a stochastic server with uncertain demand. INFORMS Journal on Computing, 25(1):116-132.

[11] Gabrel, V., Murat, C., and Thiele, A. (2014). Recent advances in robust optimization: An overview. European Journal of Operational Research, 235(3):471-483.

[12] Ge, D., Wan, G., Wang, Z., and Zhang, J. (2013). A note on appointment scheduling with piecewise linear cost functions. Mathematics of Operations Research, 39(4):1244-1251.

[13] Gul, S., Denton, B. T., Fowler, J. W., and Huschka, T. R. (2011). Bi-criteria scheduling of surgical services for an outpatient procedure center. Production and Operations Management, 20(3):406-417.

[14] Gupta, D. and Denton, B. T. (2008). Appointment scheduling in health care: Challenges and opportunities. IIE Transactions, 40(9):800-819.

[15] Jiang, R. and Guan, Y. (2016). Data-driven chance constrained stochastic program. Mathematical Programming, Series A, 158(1):291-327.

[16] Klassen, K. J. and Yoogalingam, R. (2009). Improving performance in outpatient appointment services with a simulation optimization approach. Production and Operations Management, 18(4):447-458.

[17] Kong, Q., Lee, C.-Y., Teo, C.-P., and Zheng, Z. (2013). Scheduling arrivals to a stochastic service delivery system using copositive cones. Operations Research, 61(3):711-726.

[18] Mak, H.-Y., Rong, Y., and Zhang, J. (2014). Sequencing appointments for service systems using inventory approximations. Manufacturing $\mathcal{E}$ Service Operations Management, 16(2):251262.

[19] Mak, H.-Y., Rong, Y., and Zhang, J. (2015). Appointment scheduling with limited distributional information. Management Science, 61(2):316-334.

[20] Mittal, S., Schulz, A. S., and Stiller, S. (2014). Robust Appointment Scheduling. In Jansen, K., Rolim, J. D. P., Devanur, N. R., and Moore, C., editors, Approximation, Randomization, and Combinatorial Optimization. Algorithms and Techniques (APPROX/RANDOM 2014), volume 28 of Leibniz International Proceedings in Informatics (LIPIcs), pages 356-370, Dagstuhl, Germany. Schloss Dagstuhl-Leibniz-Zentrum fuer Informatik.

[21] Pinedo, M. (2012). Scheduling: Theory, Algorithms, and Systems. Springer.

[22] Qi, J. (2016). Mitigating delays and unfairness in appointment systems. Forthcoming in Management Science.

[23] Shapiro, A. (2000). On duality theory of conic linear problems. In Semi-Infinite Programming, pages 135-165. Kluwer Academic Publishers.

[24] Zymler, S., Kuhn, D., and Rustem, B. (2013). Distributionally robust joint chance constraints with second-order moment information. Mathematical Programming, 137(1-2):167-198. 